\title{
Partitioning Inverse Monte Carlo Iterative Algorithm for Finding the Three Smallest Eigenpairs of Generalized Eigenvalue Problem
}

\author{
Behrouz Fathi Vajargah and Farshid Mehrdoust \\ Department of Statistics, Faculty of Mathematical Sciences, University of Guilan, \\ P.O. Box 1914, Rasht, Iran \\ Correspondence should be addressed to Behrouz Fathi Vajargah, fmehrdoust@guilan.ac.ir \\ Received 5 December 2010; Accepted 15 February 2011 \\ Academic Editor: Michele Benzi
}

Copyright (C) 2011 B. Fathi Vajargah and F. Mehrdoust. This is an open access article distributed under the Creative Commons Attribution License, which permits unrestricted use, distribution, and reproduction in any medium, provided the original work is properly cited.

A new Monte Carlo approach for evaluating the generalized eigenpair of real symmetric matrices will be proposed. Algorithm for the three smallest eigenpairs based on the partitioning inverse Monte Carlo iterative (IMCI) method will be considered.

\section{Introduction}

It is well known that the problem of calculating the largest or smallest generalized eigenvalue problem is one of the most important problems in science and engineering [1, 2]. This problem arises naturally in many applications. Mathematically, it is a generalization of the symmetric eigenvalue problem, and it can be reduced to an equivalent symmetric eigenvalue problem. Let $A, B \in \mathfrak{R}^{n \times n}$ be real symmetric matrices and the matrix $B$ a positive definite matrix. Consider the problem of evaluating the eigenvalues of the pencil $(A, B)$, that is, the values for which

$$
A x=\lambda B x .
$$

A generalized eigenvalue problem (1.1) is said to be symmetric positive definite (S/PD) if $A$ is symmetric and $B$ is positive definite. 


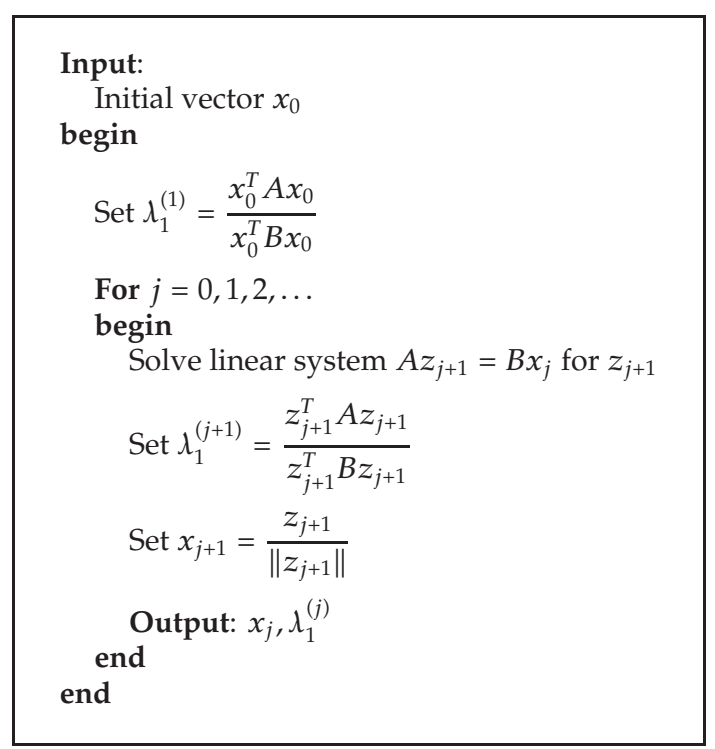

Algorithm 1

\section{Inverse Vector Iteration Method}

Another procedure for eigenvalue prediction is to use the Rayleigh quotient given by [3]

$$
\curlywedge=\mu(x)=\frac{x^{T} A x}{x^{T} B x} .
$$

since $B$ is positive definite, then (2.1) is well defined.

Theorem 2.1. Suppose that $\lambda_{\min }=\lambda_{1}<\lambda_{2} \leq \cdots \leq \lambda_{n-1}<\lambda_{n}=\lambda_{\max }$ are $n$ eigenvalues for pencil $(A, B)$ and $v_{1}, \ldots, v_{n}$, corresponding eigenvectors. Then for arbitrary initial vector $x$ one has [3]

$$
\lambda_{1}<\mu(x)<\lambda_{n}
$$

where $\mu(x)$ is as introduced in (2.1).

Theorem 2.2. The inverse vector iteration method for arbitrary choice vector $x_{0}$ is convergent to the smallest eigenvalue and corresponding eigenvector for pencil $(A, B)$. Also, the rate of convergence depends on $\mathcal{O}\left(\lambda_{2} / \lambda_{1}\right)^{k}$, where $k$ is number of iterations [3].

Algorithm 1 evaluates the smallest eigenpair based on the inverse vector iteration [4]. 


\section{Monte Carlo Method for Matrix Computations}

Suppose that the matrix $A \in \mathfrak{R}^{n \times n}$ and two vectors $f, h \in \mathfrak{R}^{n}$ are given. Consider the following Markov chain $T_{i}$ with length $i$ :

$$
T_{i}: k_{0} \longrightarrow k_{1} \cdots \longrightarrow k_{i}
$$

where for $j=1, \ldots, i, k_{j} \in\{1,2, \ldots, n\}$. The statistical nature of constructing the chain (3.1) follows as

$$
p\left(k_{0}=\alpha\right)=p_{\alpha}, \quad p\left(k_{j}=\beta \mid k_{j-1}=\alpha\right)=p_{\alpha \beta},
$$

where $p_{\alpha}$ and $p_{\alpha \beta}$ show the probability of starting chain at $\alpha$ and transition probability from state $\alpha$ to $\beta$, respectively.

In fact

$$
\sum_{\alpha=1}^{n} p_{\alpha}=1, \quad \sum_{\beta=1}^{n} p_{\alpha \beta}=1, \quad \alpha=1,2, \ldots, n
$$

Define the random variable $W_{j}$ using the following recursion for

$$
W_{0}=1, \quad W_{j}=W_{j-1} \frac{a_{k_{j-1} k_{j}}}{p_{k_{j-1} k_{j}}}, \quad j=1,2, \ldots, i
$$

Now, define the following random variable:

$$
\Theta[h]=\frac{h_{k_{0}}}{p_{k_{0}}} \sum_{j=0}^{\infty} W_{j} f_{k_{j}} .
$$

Theorem 3.1. Consider the following system:

$$
A x=b .
$$

Let the nonsingular matrix $M \in \mathfrak{R}^{n}$, such that $M A=I-L$, then the system (3.6) can be presented in the following form:

$$
x=L x+f,
$$

where $f=M b$. Then under condition $\operatorname{Max}_{i} \sum_{j=1}^{n}\left|l_{i j}\right|<1$, one has [5]

$$
E\{\Theta[h]\}=\langle h, x\rangle .
$$


Suppose that $x^{(i)}$ is the $i$ th iterative solution of the following recursion relation with $x^{(0)}=f$. If we set the random variable

$$
\Theta_{i}[h]=\frac{h_{k_{0}}}{p_{k_{0}}} \sum_{j=0}^{i} W_{j} f_{k_{j}},
$$

then

$$
E\left\{\Theta_{i}[h]\right\}=\left\langle h, x^{(i+1)}\right\rangle
$$

By simulating $N$ random paths with length $i$

$$
T_{i}^{(s)}: k_{0}^{(s)} \longrightarrow k_{1}^{(s)} \longrightarrow \cdots \longrightarrow k_{i}^{(s)}, \quad s=1,2, \ldots, N
$$

we can find

$$
\Theta_{i}^{(s)}(h)=\frac{h_{k_{0}}}{p_{k_{0}}^{(s)}} \sum_{j=0}^{i} W_{j}^{(s)} f_{k_{j}}, \quad s=1, \ldots, N
$$

The Monte Carlo estimation can be evaluated by

$$
\Theta_{i}=\frac{1}{N} \sum_{s=1}^{N} \Theta_{i}^{(s)}(h)
$$

which is an approximation of $\left\langle h, x^{(i+1)}\right\rangle$.

From all possible permissible densities, we apply the following:

$$
\begin{gathered}
p_{\alpha}=\frac{\left|h_{\alpha}\right|}{\sum_{\alpha=1}^{n}\left|h_{\alpha}\right|}, \\
p_{\alpha \beta}=\frac{\left|a_{\alpha \beta}\right|}{\sum_{\beta=1}^{n}\left|a_{\alpha \beta}\right|}, \quad \alpha=1,2, \ldots, n .
\end{gathered}
$$

The choice of the initial density vector and the transition probability matrix leads to an almost Optimal Monte Carlo (MAO) algorithm.

Theorem 3.2. Using the above choice $p=\left\{p_{\alpha}\right\}_{\alpha=1}^{n}$ and $P=\left\{p_{\alpha \beta}\right\}_{\alpha, \beta=1}^{n}$ the variance of the unbiased estimator for obtaining the inverse matrix is minimized [4].

There is a global algorithm that evaluates the solution of system (3.6) for every matrix $A$. The complexity of algorithm is $O\left(n^{2} l N\right)$, where $l$ and $N$ are the average length of Markov chian and the number of simulated paths, respectively [2]. 


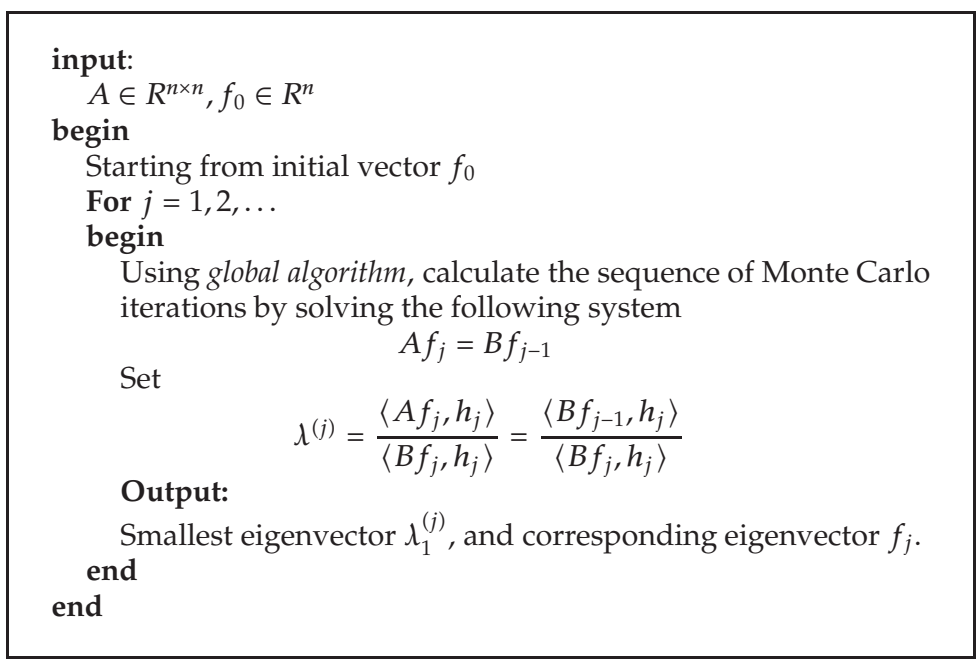

Algorithm 2

\section{Inverse Monte Carlo Iterative Algorithm (IMCI)}

Inverse Monte Carlo iterative algorithm can be applied when $A$ is a nonsingular matrix. In this method, we calculate the following functional in each steps:

$$
\frac{\left\langle A f_{j}, h_{j}\right\rangle}{\left\langle B f_{j}, h_{j}\right\rangle}=\frac{\left\langle B f_{j-1}, h_{j}\right\rangle}{\left\langle B f_{j}, h_{j}\right\rangle} .
$$

It is more efficient that we first evaluate the inverse matrix using the Monte Carlo algorithm $[1,2,4]$. The algorithm can be realized as in Algorithm 2 .

\section{Partitioning IMCI}

Let the matrix $A$ be partitioned into four blocks $A_{1}, A_{2}, A_{3}$, and $A_{4}$, where $A_{1}$ and $A_{4}$ are square matrices of order $p$ and $q$ such that $p+q=n$ :

$$
A=\left(\begin{array}{l|l}
A_{1} & A_{2} \\
\hline A_{3} & A_{4}
\end{array}\right) .
$$

By assumption that all the indicated matrix inversions are realized, it is easy to verify that

$$
A^{-1}=\left(\begin{array}{c|c}
B & L \\
\hline M & N
\end{array}\right)
$$




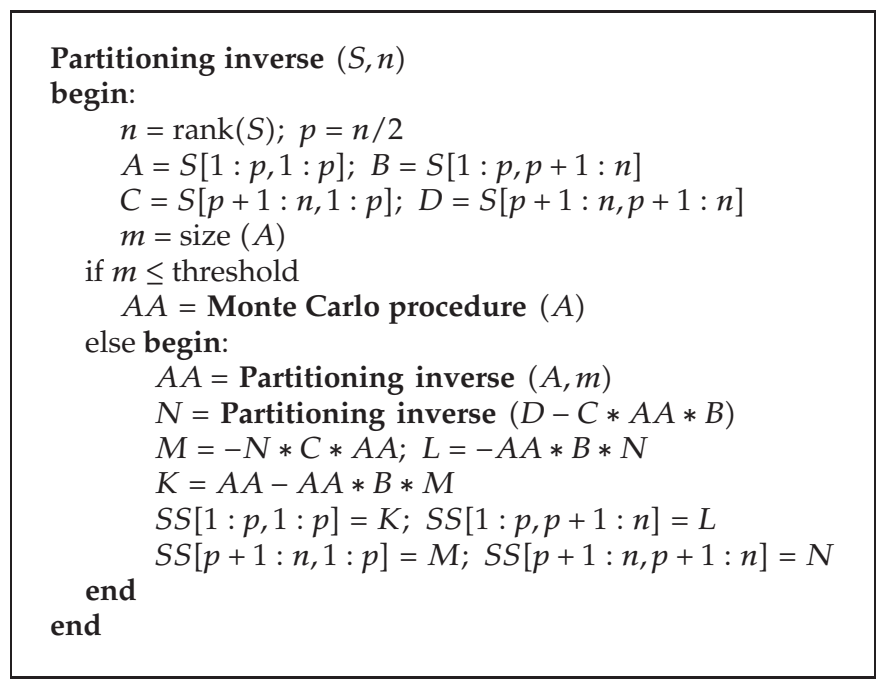

Algorithm 3

where

$$
\begin{gathered}
N=\left(A_{4}-A_{3} A_{1}^{-1} A_{2}\right)^{-1}, \quad M=-N A_{3} A_{1}^{-1}, \\
L=-A_{1}^{-1} A_{2} N, \quad K=A_{1}^{-1}-A_{1}^{-1} A_{2} M .
\end{gathered}
$$

Thus inverting a matrix of order $n$ comes down to inverting four matrices, of which two have order $p$ and two have order $q$, and several matrix multiplications. Therefore the basic Monte Carlo for solving $A f_{j}=B f_{j-1}$ will be called as the dimension of matrix Aand equals to threshold. This action causes the convergence acceleration. Now, we can use the following recursion algorithm to obtain the inverse of matrix $A$ (see Algorithm 3).

\section{Finding More Than One Generalized Eigenvalues}

Assume that an eigenvalue $\lambda_{1}$ and its corresponding eigenvector $v_{1}$ have been computed using the partitioning IMCI algorithm. In the first step of the above algorithm, we deflate the matrix $A$ to the matrix $B$. Then, we repeat again the first step of the algorithm to obtain the dominant eigenvalue of $B$ which is the second dominant eigenvalue of $A$. Let $p$ values of eigenvalues of pencil $(A, B)$ be computed. Suppose that $V_{p}$ is a matrix such that the columns of $V_{p}$ are $p$ vector of eigenvector of pencil $(A, B)$, that is,

$$
V_{p}=\left[v_{1}, \ldots, v_{p}\right]
$$

where $v_{i}$ is eigenvector corresponding eigevalue $\lambda_{i}, i=1,2, \ldots, p$. 
Table 1: Number of chains $N=80$.

\begin{tabular}{lcccccc}
\hline \multirow{2}{*}{ Dimension } & \multicolumn{3}{c}{ Eigenvalues error } & \multicolumn{3}{c}{ Eigenvectors error } \\
& $\lambda_{1}^{(1)}$ & $\lambda_{1}^{(2)}$ & $\lambda_{1}^{(3)}$ & $v_{1}^{(1)}$ & $v_{1}^{(2)}$ & $v_{1}^{(3)}$ \\
\hline $64 \times 64$ & $1.26 \times 10^{-7}$ & $4.5 \times 10^{-7}$ & $1.26 \times 10^{-6}$ & $8.36 \times 10^{-4}$ & .002813 & .008154 \\
$128 \times 128$ & $1.18 \times 10^{-7}$ & $4.46 \times 10^{-7}$ & $1.33 \times 10^{-6}$ & $6.33 \times 10^{-4}$ & .002534 & .008074 \\
$256 \times 256$ & $6.48 \times 10^{-8}$ & $4.37 \times 10^{-7}$ & $1.51 \times 10^{-6}$ & $3.16 \times 10^{-4}$ & .002151 & .008070 \\
$512 \times 512$ & $7.91 \times 10^{-8}$ & $2.87 \times 10^{-7}$ & $1.72 \times 10^{-6}$ & $1.83 \times 10^{-4}$ & .001284 & .007759 \\
$1024 \times 1024$ & $7.04 \times 10^{-8}$ & $1.99 \times 10^{-7}$ & $1.79 \times 10^{-6}$ & $1.06 \times 10^{-4}$ & .000712 & .00769 \\
\hline
\end{tabular}

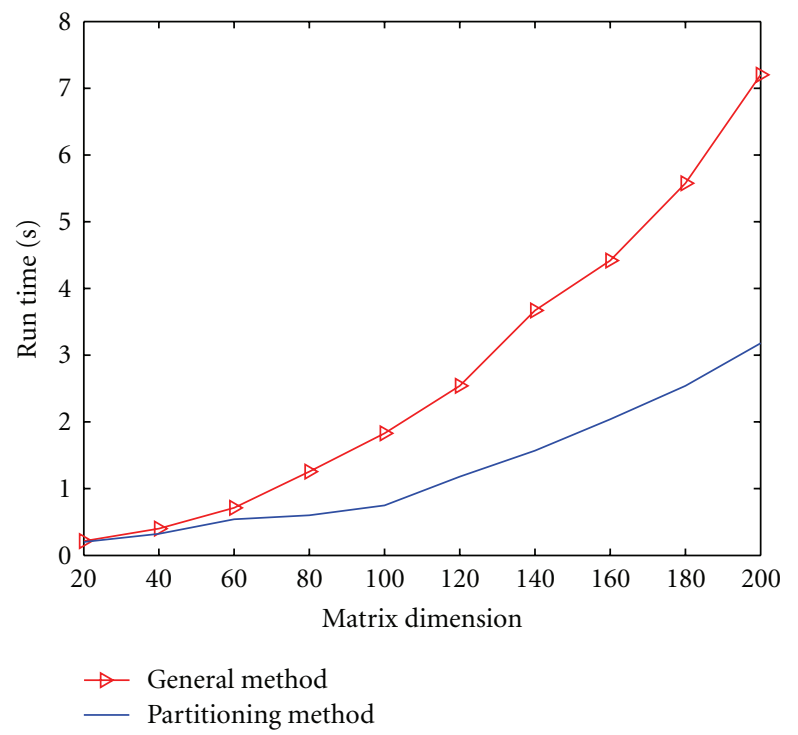

Figure 1: Computational times for general and partition methods.

Now, let

$$
\left(A_{p}, B\right)=\left(A+\left(B V_{p}\right) \Lambda\left(B V_{p}\right)^{T}, B\right)
$$

where

$$
\Lambda=\operatorname{diag}\left\{\delta_{i}-\lambda_{i}\right\}, \quad \delta_{i}>\lambda_{p}, i=1, \ldots, p
$$

Hence, if we find the $p$ th smallest eigenpair of pencil $(A, B)$, then we can evalute $\lambda_{p+1}$, that is, $(p+1)$ th smallest eigenvalue of pencil $(A, B)$.

\section{Numerical Results}

In this section, the experimental results for obtaining the three smallest eigenpairs outlined in Tables 1, 2, and 3. The numerical tests are performed on Intel(R) (Core(TM)2 CPU, 1.83 GHz) personal machine. 
Table 2: The solution when the number of chains increases.

\begin{tabular}{cccc}
\hline $\begin{array}{c}\text { Number of chains } \\
N\end{array}$ & Exact $\lambda_{1}^{(1)}=.74529395$ & Calculated eignvalues \\
\hline 20 & .74737713 & .85513704 & Exact $\lambda_{1}^{(2)}=.85537131$ \\
40 & .74529979 & .85537157 & .91008238 \\
80 & .74529407 & .85537175 & .91034304 \\
\hline
\end{tabular}

Table 3: Total computational time for general and partitioning methods.

\begin{tabular}{|c|c|c|}
\hline \multirow{2}{*}{ Dimension } & \multicolumn{2}{|c|}{ Time (Sec.) } \\
\hline & General method & Partitioning method \\
\hline $20 \times 20$ & .21 & .20 \\
\hline $40 \times 40$ & .40 & .32 \\
\hline $60 \times 60$ & .71 & .54 \\
\hline $80 \times 80$ & 1.25 & .60 \\
\hline $100 \times 100$ & 1.83 & .75 \\
\hline $120 \times 120$ & 2.54 & 1.18 \\
\hline $140 \times 140$ & 3.67 & 1.57 \\
\hline $160 \times 160$ & 4.42 & 2.04 \\
\hline $180 \times 180$ & 5.58 & 2.54 \\
\hline $200 \times 200$ & 7.20 & 3.18 \\
\hline
\end{tabular}

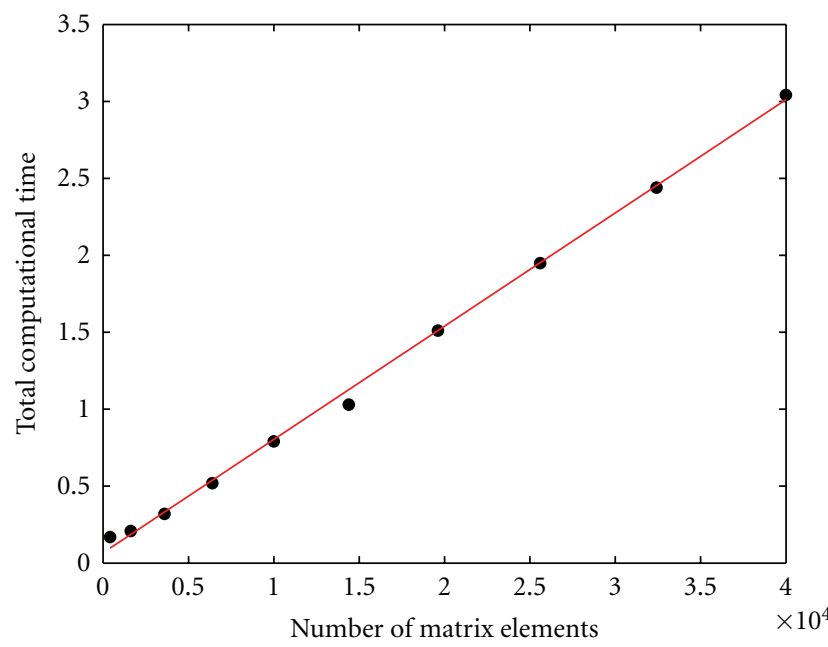

- Data

- Robust fitting curve

Figure 2: Regression function $y=0.0000658008 x+0.1048607485$.

\section{Conclusion and Future Study}

We have seen that Monte Carlo algorithms can be used for finding more than one eigenpair of generalized eigenvalue problems. We analyze the computational complexity, speedup, and efficiency of the algorithm in the case of dealing with sparse matrices. Finally, a new method 
for computing eigenpairs as the partitioned method is presented. In Figure 1 the comparison of computational times between general Monte Carlo algorithm and partitioning algorithm is shown. The scatter diagram in Figure 2, shows that there is a linear relationship between matrix dimension (equivalently, the number of matrix elements) and total computational time for partitioning IMCI.

\section{References}

[1] I. Dimov and A. Karaivanova, "Iterative Monte Carlo algorithm for linear algebra problem," Lecture Note in Computer Science, pp. 66-77, 1996.

[2] I. Dimov, Monte Carlo Methods for Applied Scientists, World Scientific Publishing, 2008.

[3] Y. Saad, Numerical Methods for Large Eigenvalue Problems, Manchester University Press, 1991.

[4] B. Fathi, "A way to obtain Monte Carlo matrix inversion with minimal error," Applied Mathematics and Computation, vol. 191, no. 1, pp. 225-233, 2007.

[5] R. Y. Rubinstein, Simulation and the Monte Carlo Method, John Wiley \& Sons, New York, NY, USA, 1981. 


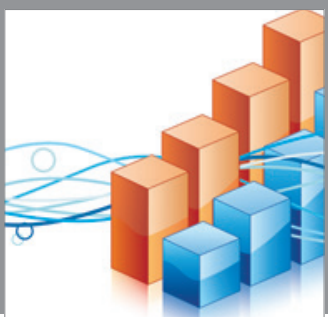

Advances in

Operations Research

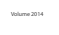

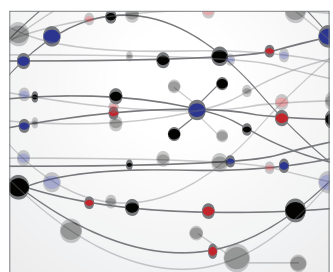

\section{The Scientific} World Journal
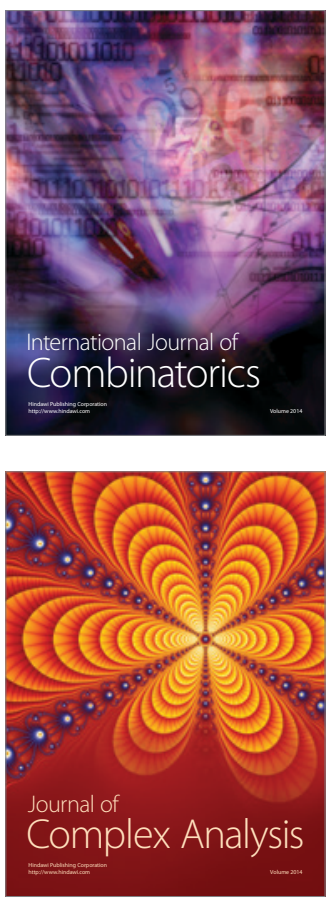

International Journal of

Mathematics and

Mathematical

Sciences
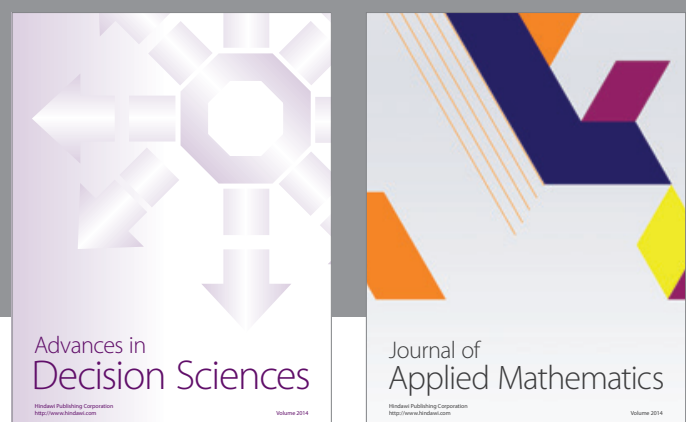

Journal of

Applied Mathematics
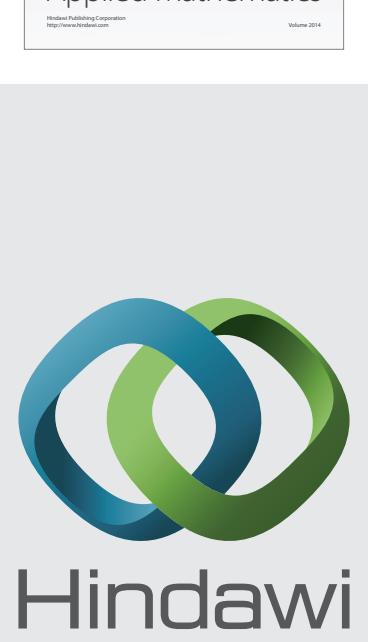

Submit your manuscripts at http://www.hindawi.com
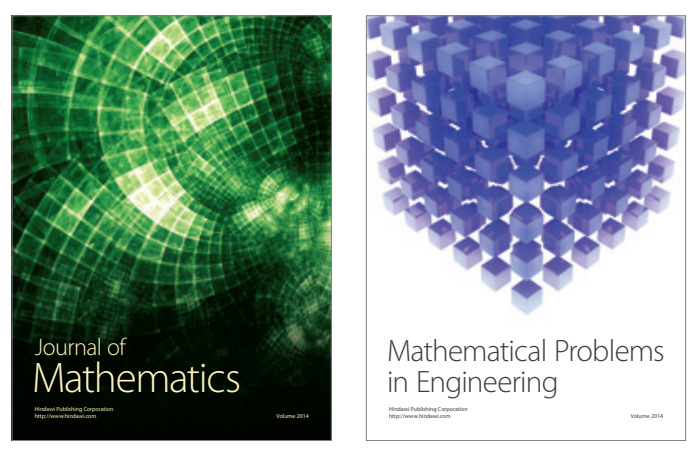

Mathematical Problems in Engineering
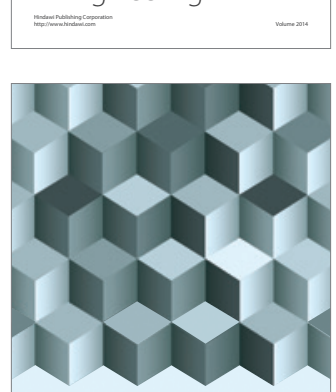

Journal of

Function Spaces
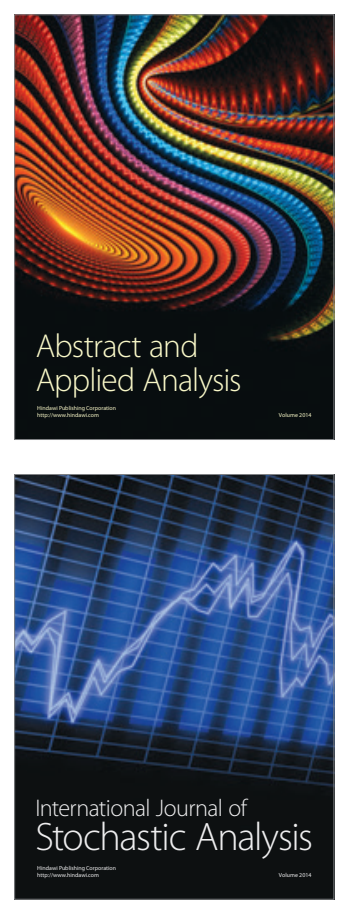

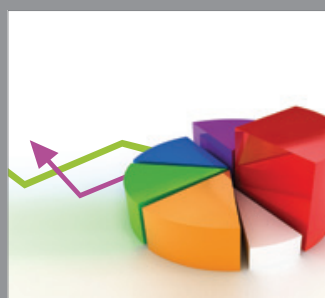

ournal of

Probability and Statistics

Promensencen
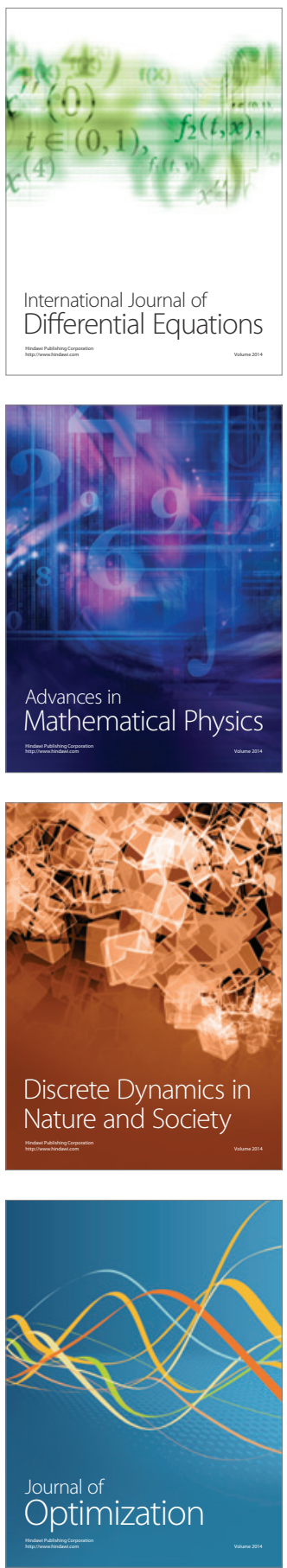